\title{
Bianchi Type - III Charged Fluid Universe in Brans-Dicke Theory of Gravitation
}

\author{
V.G. Mete ${ }^{1 *}$, K.R. Mule ${ }^{2}$,V.M. Ingle ${ }^{3}$ \\ ${ }^{1,3}$ Dept. of Mathematics, Bar. Ramrao Deshmukh Arts, Smt.Indiraji Kapadia Commerce and \\ Ny. Krishnarao Deshmukh Science College, Badnera-Amravati, India \\ ${ }^{2}$ Dept. of Mathematics, Shri. Dnyaneshwar Maskuji Burungale Science and Arts College, Shegaon, Dist.Buldana, India \\ *Corresponding Author: vmete5622@gmail.com, Tel.: +91-8956252244
}

Available online at: www.isroset.org

Received: 01/Oct/2018, Accepted: 14/Oct/2018, Online: 31/Oct/2018

\begin{abstract}
We investigate the spatially homogeneous Bianchi Type-III space time with electromagnetic field tensor and relativistic charged perfect fluid in Brans-Dicke (B-D) theory of gravity. Solutions have been obtained by using a general approach of solving the partial differential equations. It is observed that the convergent and isotropic solution of the metric function can be derived with the components of the vector potentials.
\end{abstract}

Keywords-Bianchi type-III universe, Brans-Dicke theory of gravitation, electromagnetic field, perfect fluid, vector potentials

\section{INTRODUCTION}

In recent years there has been a lot of interest in several alternative theories of gravitation; out of which the most important among them is scalar-tensor theory of gravitation formulated by Brans-Dicke [1]. This theory of gravity is one of the most competent theory due to its vast cosmological implications [2].In this theory, the scalar field has the dimensions of universe of the gravitational constant and its role is confined to its effect on gravitational field equations. This theory of gravity is mediated by a scalar field $\phi$ in addition to the usual metric tensor field $g_{i j}$ present in Einstein's theory. Among the various modifications of general relativity, the B-D theory of gravity is well known example of a scalar tensor theory in which the gravitational interaction involves a scalar field and the metric tensor.

In recent years, the study of Bianchi type models in the context of B-D theory has attracted many authors Pawar et.al [3], Sharif et.al [4], Kandalkar et.al [5], Raut et.al [6], Katore et.al [7]. A detailed discussion of B-D cosmology is given by Singh et al.[8]. Lorenz-Petzold [9] studied exact Bianchi type-III solutions in the presence of electromagnetic field. Bianchi type-I space-time in scalartensor theory have been investigated by Kumar et al.[10]. Adhav et al.[11] studied LRS Bianchi type-II cosmological model with anisotropic dark energy, Katore et al.[12,13] explored Bianchi type-V and plane symmetric space-time filled with dark energy models in B-D theory. Bianchi type III dark energy model in scalar tensor theory of gravitation explained by Naidu et al.[14]. Adhav et al. [15] explored Bianchi type-III cosmological model with negative constant deceleration parameter in B-D theory of gravity in presence of perfect fluid. Shamir et al. [16] have studied anisotropic dark energy Bianchi type-III cosmological models in B-D theory of gravity.

The Brans-Dicke field equations are given by

$$
\begin{aligned}
G_{j}^{\mu}= & \frac{-8 \pi}{\phi}\left(T_{j}^{\mu}\right)-\frac{\omega}{\phi^{2}}\left(g^{\mu i} \phi_{, i}-\frac{1}{2} g_{j}^{\mu} \phi_{, k} \phi^{, k}\right) \\
& -\frac{1}{\phi}\left(g^{\mu i} \phi_{i ; j}-g_{j}^{\mu} \phi_{; k}^{, k}\right),
\end{aligned}
$$

where $\omega$ is a dimensionless coupling constant. The function $\phi$ is known as B-D scalar field. Karade and Solanke [17] investigated Bianchi type-III universe field with the perfect fluid and scalar field coupled with electromagnetic fields in $f(R, T)$ theory of gravity. Recently Bhoyar et al.[18] discussed the Bianchi type-III and Kantowski Sachs cosmological model containing magnetic field with variable cosmological constant.

This motivates us to investigate Bianchi type-III charged fluid universe in B-D Theory of gravitation.

The paper is organized as follows: 
Section II, deals with the derivation and solutions of the field equations. A brief summary is given is section III.

\section{II.THE METRIC AND FIELD EQUATIONS}

Here, we consider a spatially homogeneous Bianchi Type-III space time in the form

$d s^{2}=-d t^{2}+A^{2} d x^{2}+B^{2} e^{-2 m x} d y^{2}+c^{2} d z^{2}$,

(1) where

$A, B$ and $C$ are functions of $t$ and $m$ is constant.

For the charged fluid, the field equations of B-D theory assume that

$$
\begin{aligned}
G_{j}^{\mu}= & \frac{-8 \pi}{\phi}\left(T_{j}^{\mu}+E_{j}^{\mu}\right)-\frac{\omega}{\phi^{2}}\left(g^{\mu i} \phi_{, i}-\frac{1}{2} g_{j}^{\mu} \phi_{, k} \phi^{, k}\right) \\
& -\frac{1}{\phi}\left(g^{\mu i} \phi_{i ; j}-g_{j}^{\mu} \phi_{; k}^{, k}\right),
\end{aligned}
$$

where $G_{j}^{\mu}$ is Einstein tensor, $E_{j}^{\mu}$ is energy momentum tensor for electromagnetic field, $T_{j}^{\mu}$ is energy momentum tensor for perfect fluid with conservation equation.

$\phi_{; k}^{k}=\frac{1}{\sqrt{-g}}\left[\sqrt{-g} \phi^{k}\right]_{, k}$

and other symbols and notations have their conventional meanings.

\section{Electromagnetic field}

The energy momentum tensor for electromagnetic field is given by

$$
E_{i j}=\frac{1}{4} F_{a b} F^{a b} g_{i j}-F_{a i} F_{b j} g^{a b},
$$

Here the electromagnetic field tensor $F_{i j}$ has the expression

$$
F_{i j}=\frac{\partial V_{i}}{\partial x^{j}}-\frac{\partial V_{j}}{\partial x^{i}},
$$

where $V_{i}$ is a four potential vector.

To achieve the compatibility with space time (1), we assume electromagnetic vector potential as

$$
V_{i}=\left[\alpha(x) v_{1}(t), v_{2}(t), v_{3}(t), v_{4}(t)\right],
$$

Noting (4) and (5) we can deduce easily the following

$$
F_{14}=\alpha \dot{v}_{1}, F_{24}=\dot{v}_{2}, F_{34}=\dot{v}_{3}, F_{43}=-\dot{v}_{3},
$$

From equations (4), (5) and (6), we can deduce

$$
F_{a b} F^{a b}=-2\left[\frac{\alpha^{2} \dot{v}_{1}^{2}}{A^{2}}+\frac{\dot{v}_{2}^{2}}{B^{2} e^{-2 m x}}+\frac{\dot{v}_{3}^{2}}{C^{2}}\right],
$$

Using (3) we can deduce the components of energy momentum tensors

$$
\begin{aligned}
& E_{1}^{1}=\frac{1}{2} \frac{\alpha^{2} \dot{v}_{1}^{2}}{A^{2}}-\frac{1}{2} \frac{\dot{v}_{2}^{2}}{B^{2} e^{-2 m x}}-\frac{1}{2} \frac{\dot{v}_{3}^{2}}{C^{2}}, \\
& E_{2}^{1}=\frac{\alpha \dot{v}_{1} \dot{v}_{2}}{A^{2}}, \\
& E_{3}^{1}=\frac{\alpha \dot{v}_{1} \dot{v}_{3}}{A^{2}}, \\
& E_{2}^{2}=-\frac{1}{2} \frac{\alpha^{2} \dot{v}_{1}^{2}}{A^{2}}+\frac{1}{2} \frac{\dot{v}_{2}^{2}}{B^{2} e^{-2 m x}}-\frac{1}{2} \frac{\dot{v}_{3}^{2}}{C^{2}}, \\
& E_{3}^{2}=\frac{\dot{v}_{2} \dot{v}_{3}}{B^{2} e^{-2 m x}}, \\
& E_{1}^{3}=\frac{\alpha \dot{v}_{1} \dot{v}_{3}}{C^{2}}, \\
& E_{3}^{3}=-\frac{1}{2} \frac{\alpha^{2} \dot{v}_{1}^{2}}{A^{2}}-\frac{1}{2} \frac{\dot{v}_{2}^{2}}{B^{2} e^{-2 m x}}+\frac{1}{2} \frac{\dot{v}_{3}^{2}}{C^{2}}, \\
& E_{4}^{4}=\frac{1}{2} \frac{\alpha^{2} \dot{v}_{1}^{2}}{A^{2}}+\frac{1}{2} \frac{\dot{v}_{2}^{2}}{B^{2} e^{-2 m x}}+\frac{1}{2} \frac{\dot{v}_{3}^{2}}{C^{2}},
\end{aligned}
$$

The stress energy tensor of a perfect fluid with density $\rho$, pressure $p$ and four velocity $u_{i}$ is given by

$$
T_{j}^{i}=(\rho+p) u^{i} u_{j}-p \delta_{i}^{j},
$$

where $g_{i j} u^{i} u^{j}=1$

For co-moving coordinate system, we have

$u_{x}=0, u_{y}=0, u_{z}=0, u_{t} \neq 0$,

Accordingly (9) provides

$$
\begin{aligned}
& T_{1}^{1}=(\rho+p) u^{1} u_{1}-p \delta_{1}^{1}=-p, \\
& T_{2}^{2}=(\rho+p) u^{2} u_{2}-p \delta_{2}^{2}=-p, \\
& T_{3}^{3}=(\rho+p) u^{3} u_{3}-p \delta_{3}^{3}=-p,
\end{aligned}
$$


$T_{4}^{4}=(\rho+p) u^{4} u_{4}-p \delta_{4}^{4}=\rho$,

$T_{1}^{1}+E_{1}^{1}=\frac{1}{2} \frac{\alpha^{2} \dot{v}_{1}^{2}}{A^{2}}-\frac{1}{2} \frac{\dot{v}_{2}^{2}}{B^{2} e^{-2 m x}}-\frac{1}{2} \frac{\dot{v}_{3}^{2}}{C^{2}}-p$,

$T_{2}^{1}+E_{2}^{1}=\frac{\alpha \dot{v}_{1} \dot{v}_{2}}{A^{2}}$,

$T_{3}^{1}+E_{3}^{1}=\frac{\alpha \dot{v}_{1} \dot{v}_{3}}{A^{2}}$,

$T_{2}^{2}+E_{2}^{2}=-\frac{1}{2} \frac{\alpha^{2} \dot{v}_{1}^{2}}{A^{2}}+\frac{1}{2} \frac{\dot{v}_{2}^{2}}{B^{2} e^{-2 m x}}-\frac{1}{2} \frac{\dot{v}_{3}^{2}}{C^{2}}-p$,

$T_{3}^{2}+E_{3}^{2}=\frac{\dot{v}_{2} \dot{v}_{3}}{B^{2} e^{-2 m x}}$,

$T_{3}^{3}+E_{3}^{3}=-\frac{1}{2} \frac{\alpha^{2} \dot{v}_{1}^{2}}{A^{2}}-\frac{1}{2} \frac{\dot{v}_{2}^{2}}{B^{2} e^{-2 m x}}+\frac{1}{2} \frac{\dot{v}_{3}^{2}}{C^{2}}-p$,

$T_{4}^{4}+E_{4}^{4}=\frac{1}{2} \frac{\alpha^{2} \dot{v}_{1}^{2}}{A^{2}}+\frac{1}{2} \frac{\dot{v}_{2}^{2}}{B^{2} e^{-2 m x}}+\frac{1}{2} \frac{\dot{v}_{3}^{2}}{C^{2}}+\rho$,

Conservation Law is

$\frac{\partial}{\partial x^{i j}}\left(\sqrt{-g} F^{i j}\right)=0$,

This equation with different combination of $i$ and $j$, gives following equations

$\left[\frac{\dot{v}_{1}}{v_{1}}\right]+\frac{\dot{v}_{1}^{2}}{v_{1}^{2}}+\frac{\dot{v}_{1}}{v_{1}}\left[\frac{\dot{B}}{B}+\frac{\dot{C}}{C}-\frac{\dot{A}}{A}\right]=0$,

$\left[\frac{\dot{v}_{2}}{v_{2}}\right]+\frac{\dot{v}_{2}^{2}}{v_{2}^{2}}+\frac{\dot{v}_{2}}{v_{2}}\left[\frac{\dot{A}}{A}+\frac{\dot{C}}{C}-\frac{\dot{B}}{B}\right]=0$,

$\left[\frac{\dot{v}_{3}}{v_{3}}\right]+\frac{\dot{v}_{3}^{2}}{v_{3}^{2}}+\frac{\dot{v}_{3}}{v_{3}}\left[\frac{\dot{A}}{A}+\frac{\dot{B}}{B}-\frac{\dot{C}}{C}\right]=0$,

$\phi_{; k}^{, k}=-\ddot{\phi}-\ddot{\phi}\left[\frac{\dot{A}}{\mathrm{~A}}+\frac{\dot{B}}{\mathrm{~B}}+\frac{\dot{C}}{C}\right]$,

From the vanishing components of Einstein tensor, using equations (2) and (4), we deduce

$\frac{\dot{v}_{1} \dot{v}_{2}}{v_{1} v_{2}}=\frac{\dot{v}_{1} \dot{v}_{3}}{v_{1} v_{3}}=\frac{\dot{v}_{2} \dot{v}_{3}}{v_{2} v_{3}}=0$
$\frac{\dot{v}_{1}}{v_{1}}=\frac{\dot{v}_{2}}{v_{2}}=\frac{\dot{v}_{3}}{v_{3}}=\frac{\dot{D}}{D}$,

where $D$ is an unknown function of $t$

Integrating this with respect to $t$, we get

$v_{1}=k_{1} D \quad, v_{2}=k_{2} D \quad, \quad v_{3}=k_{3} D$

where $k_{1} k_{2}$ and $k_{3}$ are constants

Inserting (12) in (11), we get

$\left(\frac{\dot{D}}{D}\right)^{2}=0$,

With the aid of equation (12), we can write the equation (10) as,

$\left(\frac{\dot{D}}{D}\right)+\left(\frac{\dot{D}}{D}\right)^{2}+\frac{\dot{D}}{D}\left(\frac{\dot{B}}{\mathrm{~B}}+\frac{\dot{C}}{C}-\frac{\dot{A}}{\mathrm{~A}}\right)=0$,

$\left(\frac{\dot{D}}{D}\right)+\left(\frac{\dot{D}}{D}\right)^{2}+\frac{\dot{D}}{D}\left(\frac{\dot{A}}{A}+\frac{\dot{C}}{C}-\frac{\dot{B}}{B}\right)=0$,

$\left(\frac{\dot{D}}{D}\right)+\left(\frac{\dot{D}}{D}\right)^{2}+\frac{\dot{D}}{D}\left(\frac{\dot{A}}{A}+\frac{\dot{B}}{B}-\frac{\dot{C}}{C}\right)=0$

From equations (15a), (15b)and (15c), we have

$\frac{\dot{A}}{A}=\frac{\dot{B}}{B}=\frac{\dot{C}}{C}$,

Integrating with respect to $t$, we get

$A=k_{4} B, \quad B=k_{5} C, \quad C=k_{6} A$,

where $k_{4}, k_{5}$ and $k_{6}$ are constants.

We attempt to express the component of $T_{j}^{i}$ in terms of $T_{4}^{4}$ for this consider the expression

$$
\begin{aligned}
& \frac{\alpha^{2} \dot{v}_{1}^{2}}{A^{2}}+\frac{\dot{v}_{2}^{2}}{B^{2} e^{-2 m x}}+\frac{\dot{v}_{3}^{2}}{C^{2}} \\
& =\left[\frac{\alpha^{2} v_{1}^{2}}{A^{2}}+\frac{v_{2}^{2}}{B^{2} e^{-2 m x}}+\frac{v_{3}^{2}}{C^{2}}\right]\left(\frac{\dot{D}}{D}\right)^{2}=0 \\
& T_{4}^{4}=\frac{1}{2} \frac{\alpha^{2} \dot{v}_{1}^{2}}{A^{2}}+\frac{1}{2} \frac{\dot{v}_{2}^{2}}{B^{2} e^{-2 m x}}+\frac{1}{2} \frac{\dot{v}_{3}^{2}}{C^{2}}+\rho=\rho, \\
& T_{1}^{1}=-T_{4}^{4}+\rho-p,
\end{aligned}
$$


$T_{2}^{2}=-T_{4}^{4}+\rho-p$,

$T_{3}^{3}=-T_{4}^{4}+\rho-P$,

Now, considering the non-vanishing component of Einstein tensor, from equation (2), we derive

$$
\begin{aligned}
\frac{\ddot{B}}{B}+\frac{\ddot{C}}{C}+\frac{\dot{B} \dot{C}}{B C}= & \frac{-8 \pi}{\phi}\left[-T_{4}^{4}+\rho-p\right] \\
& -\frac{1}{2} \omega\left(\frac{\dot{\phi}}{\phi}\right)^{2}-\frac{\ddot{\phi}}{\phi}-\frac{\dot{\phi}}{\phi}\left(\frac{\dot{B}}{B}+\frac{\dot{C}}{C}\right),
\end{aligned}
$$$$
\frac{\ddot{A}}{A}+\frac{\ddot{C}}{C}+\frac{\dot{A} \dot{C}}{A C}=-\frac{8 \pi}{\phi}\left[-T_{4}^{4}+\rho-p\right]-\frac{1}{2} \omega\left(\frac{\dot{\phi}}{\phi}\right)^{2}
$$$$
-\frac{\ddot{\phi}}{\phi}-\frac{\dot{\phi}}{\phi}\left(\frac{\dot{A}}{A}+\frac{\dot{C}}{C}\right)
$$$$
-\frac{m^{2}}{A^{2}}+\frac{\ddot{A}}{A}+\frac{\ddot{B}}{B}+\frac{\dot{A} \dot{B}}{A B}=-\frac{8 \pi}{\phi}\left[-T_{4}^{4}+\rho-p\right]
$$$$
-\frac{1}{2} \omega\left(\frac{\dot{\phi}}{\phi}\right)^{2}-\frac{\ddot{\phi}}{\phi}-\frac{\dot{\phi}}{\phi}\left(\frac{\dot{A}}{A}+\frac{\dot{B}}{B}\right),
$$$$
-\frac{m^{2}}{A^{2}}+\frac{\dot{A} \dot{B}}{A B}+\frac{\dot{B} \dot{C}}{B C}+\frac{\dot{A} \dot{C}}{A C}=-\frac{8 \pi}{\phi}[\rho]+\frac{1}{2} \omega\left(\frac{\dot{\phi}}{\phi}\right)^{2}
$$$$
-\frac{\dot{\phi}}{\phi}\left(\frac{\dot{A}}{A}+\frac{\dot{B}}{B}+\frac{\dot{C}}{C}\right) \text {. }
$$

$\frac{\dot{A}}{A}-\frac{\dot{B}}{B}=0$,

Integrating $(19 \mathrm{e})$ with respect to $t$, we get

$A=k_{7} B$,

where $k_{7}$ is constant.

From equations (19a) and (19b), we get

$$
\frac{\ddot{B}}{B}-\frac{\ddot{A}}{A}+\frac{\ddot{C}}{C}\left(\frac{\dot{B}}{B}-\frac{\dot{A}}{A}\right)+\frac{\dot{\phi}}{\phi}\left(\frac{\dot{B}}{B}-\frac{\dot{A}}{A}\right)=0
$$

From equations (19b) and (19c), we get

$$
\frac{m^{2}}{A^{2}}+\frac{\ddot{C}}{C}-\frac{\ddot{B}}{B}+\frac{\dot{A}}{A}\left[\frac{\dot{C}}{C}-\frac{\ddot{B}}{B}\right]+\frac{\dot{\phi}}{\phi}\left[\frac{\dot{C}}{C}-\frac{\dot{B}}{B}\right]=0,
$$

Using equations (19c) and (19a), we obtain

$$
-\frac{m^{2}}{A^{2}}+\frac{\ddot{A}}{A}-\frac{\ddot{C}}{C}+\frac{\dot{B}}{B}\left[\frac{\dot{A}}{A}-\frac{\dot{C}}{C}\right]+\frac{\dot{\phi}}{\phi}\left[\frac{\dot{A}}{A}-\frac{\dot{C}}{C}\right]=0,
$$

Eliminating $\frac{m^{2}}{A^{2}}$ between (20b) \& (20c), we get

$$
\begin{aligned}
& \frac{\ddot{A}}{A}-\frac{\ddot{B}}{B}+\frac{\dot{A} \dot{C}}{A C}-\frac{\dot{B} \dot{C}}{B C}+\frac{\dot{\phi}}{\phi}\left(\frac{\dot{A}}{A}-\frac{\dot{B}}{B}\right)=0, \\
& \frac{\ddot{A}}{A}-\frac{\ddot{B}}{B}+\frac{\dot{C}}{C}\left[\frac{\dot{A}}{A}-\frac{\dot{B}}{B}\right]+\frac{\phi}{\phi}\left[\frac{\dot{A}}{A}-\frac{\dot{B}}{B}\right]=0,
\end{aligned}
$$

Upon integration of (20a) and (20e), yields

$$
\frac{A}{B}=k_{9} \exp \left\{k_{8} \int \frac{1}{A B C \phi} d t\right\}
$$

Similarly

$\frac{B}{A}=k_{11} \exp \left\{k_{10} \int \frac{1}{A B C \phi} d t\right\}$,

We can express the values of $A$ and $B$ in the following form

$$
\begin{aligned}
& A=(A B C)^{1 / 3} k_{12} \exp \left\{k_{11} \int \frac{1}{A B C \phi} d t\right\}, \\
& B=(A B C)^{1 / 3} k_{14} \exp \left\{k_{13} \int \frac{1}{A B C \phi} d t\right\},
\end{aligned}
$$

Equation (17) implies $C$ is scalar multiple of $A$

$$
C=(A B C)^{1 / 3} k_{16} \exp \left\{k_{15} \int \frac{1}{A B C \phi} d t\right\},
$$

Using equations (15) and (22), we get,

$$
\frac{\ddot{D}}{\dot{D}}+\frac{\dot{A}}{A}=0 \text {, }
$$

Integrating above equation, we get

$D=k_{17} \int \frac{1}{A} d t+k_{18}$,

Using (24) the equation (13) reduces to

$$
v_{1}=k_{19} \int \frac{1}{A} d t+k_{20},
$$

$v_{2}=k_{21} \int \frac{1}{A} d t+k_{22}$,

$v_{3}=k_{23} \int \frac{1}{A} d t+k_{24}$, 
$v_{4}$ is undetermined.

The metric in (1), with the help of (22) can be redefined in the form

$$
\begin{aligned}
d s^{2}= & (A B C)^{2} / 3\left[K^{\prime} \exp K^{\prime \prime} \int \frac{1}{(A B C) \phi} d t\right]^{2}\left(d x^{2}+e^{-2 m x} d y^{2}+d z^{2}\right) \\
& -d t^{2},
\end{aligned}
$$

where $K^{\prime}=k_{12} k_{14,} k_{16}$ and $K^{\prime \prime}=k_{11} k_{13,} k_{15}$

are constants.

\section{CONCLUSION}

In this present paper, we have presented Bianchi Type-III space time with electromagnetic field tensor and relativistic charged perfect fluid in the context of Brans-Dicke theory of gravity. We have derived and solved the gravitational field equations corresponding to B-D theory. It is observed that the convergent, non-singular, isotropic solutions can be obtained along with the components of vector potential. It is also interesting to note that the investigated models are free from singularity.

\section{ACKNOWLEDGMENT}

Authors are grateful to the anonymous referee and editor for imparting valuable suggestions which have enabled us to improve the manuscript.

\section{REFERENCES}

[1] C. Brans and R. H. Dicke, "Mach's Principle and a Relativistic Theory of Gravitation",Phys.Rev. 124,pp.925, 1961.

[2] Orfeu Bertolami and P. J. Martins, "Non minimal coupling and quintessence",Phys. Rev. D 61, 64007,2000.

[3] D.D. Pawar, S.N. Bayaskar, V.R. Patil,"Plane Symmetric Cosmological Model with Thick Domain Walls in Brans-Dicke Theory of Gravitation", Bulg. J. Phys. 36 pp. 68-75, 2009.

[4] Sharif M and SairaWaheed, "Anisotropic Universe Models in Brans-Dicke Theory”, Eur. Phys. J. C72 pp.1876, 2012.

[5] Sharad Kandelkar, Seema Samdurkar," Bianchi Type-V Cosmological Model with Linear quation of State in BransDicke Theory of Gravitation", Int.J.of Astronomy and Astrophysics,4, pp.429-436, 2014.

[6] V.B.Raut, K.S.Adhav,S.D.Katore, S.D. and N.K. Sarkate,"Magnetized Anisotropic Dark Energy Bianchi Type-III Cosmological Models in Brans-Dicke Theory of Gravitation" ,Int.J. of Advanced Applied Physics Research, 1, pp.30-38,2014.

[7] Shivdas. D. Katore, A. Y. Shaikh, N. K. Sarkate,G. B. Tayade,"Dynamics of Bianchi type-III Universe with Magnetized Anisotropic Dark Energy",Prespacetime Journal, Vol. 3 , Issue 2 ,pp. 154-169,2012.

[8] T. Singh L. N. Rai ,Tarkeshwar Singh,"Anisotropic cosmological model in Brans-Dicke heory" ,Asrtophys Space Sci., Vol.96, issue 1, pp.95-105, 1983.
[9] Lorenz-Petzold , D., "Tilted electromagnetic Bianchi type III cosmological solution",Astrophys. Space Sci., 85, pp.59 61,1982 .

[10] Suresh Kumar ,C. P. Singh,"Exact Bianchi Type-I Cosmological Models in a Scalar-tensor Theory", Int. J. Theor. Phys., Vol.47, Issue 6, pp. 1722-1730,2008.

[11] K.S. Adhav, "LRS Bianchi Type-II Cosmological Models with Anisotropic Dark Energy", Electronic J.Theor. Phys , 9, No. 26, pp.239-250,2012.

[12] Shivdas. D. Katore, \& A. Y. Shaikh, "Kantowski-Sachs Dark Energy Model in f(R,T) Gravity", Prespacetime J., Vol. 3(11), pp.1087-1096, 2012.

[13] S.D.Katore, A.Y. Shaikh, "Plane Symmetric Dark Energy Model in Brans-Dicke Theory of Gravitation", Bulg. J. Phys. vol.39 no.3, pp. 241-247,2012.

[14] R.L.,Naidu, B. Satyanarayana and D.R.K.Reddy,"Bianchi TypeIII Dark Energy Model in a Saez-Ballester Scalar-Tensor Theory", Int. J. Theor. Phys., Vol.51, pp. 2857-2862, 2012

[15] K.S. Adhav, A.S, Nimkar, M.R, Ugale, M.V.Dawande,"Bianchi Type-III Cosmological Model with Negative Constant Deceleration Parameter in Brans Dicke Theory of Gravitation", Int. J Theor.Phys.,Vol. 47, Issue 3, pp 634-639, 2008.

[16] M.F.Shamir, A.A. Bhatti, "Anisotropic Dark Energy Bianchi Type III Cosmological Models in Brans Dicke Theory of Gravity", Canadian Journal of Physics, Vol.90(2) pp.193-198, 2012.

[17] D.T.Solanke,T.M. Karade,“ Bianchi Type-III Universe Filled with Combination of Perfect Fluid and Scalar Field Coupled with Electromagnetic Fields in $\mathrm{f}(\mathrm{R}, \mathrm{T})$ Theory of Gravity " ,Int.Journal of Mathematical Archive,7 (7), pp.151-162,2016.

[18] S.R.Bhoyar, V.R.Chirde, “ Magnetized Anti-stiff fluid Cosmological Models with Variable Cosmological constant", Int.J. Scientific Research in Mathematical and Statistical Science, 5 (1),pp.11-18,2018.

\section{AUTHORS PROFILE}

Dr. Vijay G. Mete pursed M.Sc., M.Phil., Ph.D. from Sant Gadge BabaAmravati University, Amravati, India.He is currently working as a AssociateProfessor in Department of Mathematics,R.D.I.K \& K.D. College, BadneraAmravati. Presently he is a member of Board of Studies, S.G.B. Amravati University, Amravati. He has published more than 60 research papers in international refereed journals and completed two minor research projects funded by UGC. His main research work focuses on relativity, theories of gravitation and cosmology.He has 26 years of teaching experience and 18 years of research experience.

Mr. K.R.Muke, pursed M.Sc., M.Phil.He is currently pursuing Ph.D. andworking as a Assistant Professor at S.D.M.B.Science \& Arts College, Shegaon.

Mr.V.M.Ingle, pursed M.Sc., He is a recipient of Joint CSIR/JRF fellowship awarded by UGC, New Delhi, India vide Sr.No.2061641218 Ref. No. 19/06/2016(i) EU-V dt.28/02/2017.
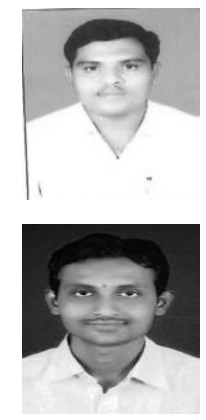